\title{
AN INTRODUCTION TO COMPACT LIE GROUPS
}




\section{SERIES IN PURE MATHEMATICS}

Editor: C C Hsiung

Associate Editors: S S Chern, S Kobayashi, I Satake, Y-T Siu, W-T Wu and $M$ Yamaguti

\section{Part I. Monographs and Textbooks}

Volume 1: Total Mean Curvature and Submanifolds on Finite Type $B$ YChen

Volume 3: Structures on Manifolds $K$ Yano \& MKon

Volume 4: Goldbach Conjecture Wang Yuan (editor)

Volume 6: Metric Rigidity Theorems on Hermitian Locally Symmetric Manifolds Ngaiming Mok

Volume 7: The Geometry of Spherical Space Form Groups Peter B Gilkey

Volume 9: Complex Analysis TO Moore \& E H Hadlock

Volume 10: Compact Riemann Surfaces and Algebraic Curves Kichoon Yang

\section{Part II. Lecture Notes}

Volume 2: A Survey of Trace Forms of Algebraic Number Fields PE Conner \& A Perlis

Volume 5: Measures on Infinite Dimensional Spaces $Y$ Yamasaki

Volume 8: Class Number Parity $P E$ Conner \& J Hurrelbrink

Volume 11: Topics in Mathematical Analysis Th M Rassias (editor)

Volume 12: A Concise Introduction to the Theory of Integration Daniel W Stroock 


\section{Series in Pure Mathematics - Volume 13}

\section{INTRODUCTION TO

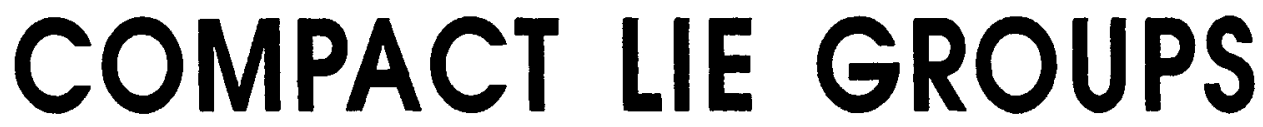

\section{Howard D Fegan}

Department of Mathematics and Statistics University of New Mexico USA 


\section{Published by}

World Scientific Publishing Co. Pre. Lid.

P O Box 128, Farrer Road, Singapore 9128

USA office: Suite 1B, 1060 Main Street, River Edge, NJ 07661

UK office: 73 Lynton Mead, Totueridge, London N20 8DH

Library of Congress Cataloging-in-Publication data is available.

\section{AN INTRODUCTION TO COMPACT LIE GROUPS \\ Copyright 1991 by World Scientific Publishing Co. Pte. Ltd.}

All rights reserved. This book, or parts thereof, may not be reproduced in any form or by any means, electronic or mechanical, including photocopying, recording or any information storage and retrieval system now known or to be invented, without written permission from the Publisher.

ISBN $981-02-0702-6$

Printed in Singapare by JBW Printers \& Binders Pte. Ltd. 
For Ann 
This page is intentionally left blank 


\section{Contents}

Preface $\quad$ ix

1 Calculus on Manifolds 1

2 Groups and Lie Groups $\quad 7$

3 One-Parameter Subgroups and the Exponential Map 15

4 The Campbell-Baker-Hausdorff Formula 25

5 The Adjoint Representation 33

6 Maximal Tori 43

7 Representation Theory $\quad 61$

8 Roots and Weights $\quad 69$

9 Weyl's Formulae $\quad 83$

10 Differential Operators on Compact Lie Groups 93

11 The Riemannian Geometry of a Compact Lie Group 99

12 The Trace of the Heat Kernel 105

Appendix 1: The Tensor Product 111

Appendix 2: Clifford Algebras and the Spin Groups 115

Solutions and Hints for Selected Exercises 119

$\begin{array}{ll}\text { Bibliography } & 127\end{array}$

$\begin{array}{ll}\text { Index } & 129\end{array}$ 
This page is intentionally left blank 


\section{Preface}

This book grew out of a seminar held at the University of New Mexico. Its aim is to provide the reader with the tools to do analysis on Lie groups. Of course, much standard material is presented which can be used for many different purposes. It is intended to provide the beginning graduate student with access to the subject of compact Lie groups. However, the material should also be suitable for senior undergraduates who are willing to spend the time that the subject requires. The other segment of the intended audience is that of research workers in other fields.

The subject of compact Lie groups is a rather old subject which is enjoying renewed attention. Since it is fairly old there has been time for the results to mature. Thus, there is a good compromise between stating results in great generality, and so losing sight of the details, or conversely in becoming too deeply embedded in details. It is this maturity which makes the subject ideal as an introduction to advanced mathematics. The other reason for the renewal of interest is the wide area of applications. The most outstanding example of this comes from gauge theory in physics. Here the mathematical model of the physical situation involves a principal fibre bundle with a compact Lie group as fibre.

A key feature of the book is the mixture of abstract theory and concrete example. In fact, there was a general plan for each chapter: to start with, a discussion of the theory, and then illustrate it in the case of some of the classical matrix groups. Not every chapter naturally fits such a plan, and when this happens, the plan has been violated so that the mathematics can appear as naturally as possible. The most obvious exception is the first chapter. As a consequence of this procedure, we have given a detailed explicit description of the structure, representation theory, and differential geometry of the vast majority of compact Lie groups, which most readers are likely to encounter in practice.

Now, for some detailed comments on the contents. A Lie group is both a group and a manifold. In Chapter 1, we study the basic theory of manifolds. Due to the special nature of compact Lie groups, relatively little of the theory of manifolds is actually needed. The discussion in Chapter 1 is generally restricted to such theory as is necessary to study Lie groups and describe the context of this theory. 
Chapters two, three, and four give the basic structure of compact Lie groups. In Chapter 2, we give the basic definitions. In Chapter 3, we see the Lie algebra come into play. Lie algebras are, generally, easier to work with than Lie groups. The exponential map enables us to pass between the group and algebra. In fact, on a suitably small part of the Lie algebra, the exponential map provides us with an isomorphism with part of the group. Chapter 4 discusses the CampbellBaker-Hausdorff formula, which shows one major manifestation of the nonabelian nature of some Lie groups.

The transition between studying the structure of compact Lie groups and their representation theory is in chapters five and six. In Chapter 5, we meet the adjoint representation, which is obtained from letting a group act on itself. This is used in Chapter 6 when we show that any two maximal tori are conjugate. This is a major result about the structure of compact Lie groups, which in turn will be used to describe the representations of such groups.

We are now at a point where we can study representation theory. This is the subject of chapters seven, eight, and nine. In Chapter 7, we give the basic definitions. It is an amazing fact that one can explicitly describe all the representations of a compact Lie group. The classification is by highest weights, and is given in Chapter 8 . There are some remarkable formulae due to Hermann Weyl, which are established in Chapter 9.

The final three chapters are about analysis on a compact Lie group. They all use representation theory quite heavily. In Chapter 10, we describe the invariant differential operators on a compact Lie group. Chapter 11 gives the Levi-Civita connection and the various curvatures. To illustrate the use of this, we study the trace of the heat kernel in Chapter 12. This includes a proof of the "strange formula" of Freudenthal and deVries.

There are two appendices. The first is about the tensor product and explains some multilinear algebra. In the second, we define Clifford algebras and give a construction of the spin groups.

Finally, one should note that each chapter finishes with a set of exercises. Working exercises is one of the main ways of learning mathematics and they are commended to the reader.

There are acknowledgements to be made. I have learned this material in the course of many years and from many sources. It is impossible to list all of the mathematicians who have contributed to my knowledge on the subject. The material in these notes is well-known, although the point of view is perhaps fresh, and is mainly drawn from 
the books in the bibliography. All of these references have been used. In at least one case, the proof of a theorem is the result of combining three different proofs from these references. This is why we have chosen to make a general acknowledgement rather than give specific references at each point in the text. 
This page is intentionally left blank 


\begin{abstract}
Correct method of reckoning, for grasping the meaning of things and knowing everything that is, obscurities ... and all secrets.
\end{abstract}

Ahmose 16th Century B.C.

This is an English rendering of the title of an Egyptian papyrus. It is taken from the book:

Gay Robins and Charles Shute, "The Rhind Mathematical Papyrus," British Museum Publications, London, 1987. Quoted by kind permission of the Trustees of the British Museum. 